\title{
Molecular characterization and tissue-specific expression of invariant chain in the muscovy duck (Cairina moschata)
}

\author{
S.J. Liu ${ }^{1,2}$, F.F. Chen ${ }^{1}$, Y. Dai ${ }^{1}$, C. Wu ${ }^{1}$, Q.S. $\mathrm{Ni}^{1}$ and W.Y. Yu ${ }^{1}$ \\ ${ }^{1}$ Key Laboratory of Zoonoses of Anhui Province, \\ Anhui Agricultural University, Hefei, China \\ ${ }^{2}$ School of Life Science, Fuyang Teachers' College of Anhui Province, \\ Fuyang, China \\ Corresponding author: W.Y. Yu \\ E-mail: yuweiyi@ahau.edu.cn
}

Genet. Mol. Res. 10 (4): 2867-2880 (2011)

Received February 2, 2011

Accepted May 16, 2011

Published November 22, 2011

DOI http://dx.doi.org/10.4238/2011.November.22.1

\begin{abstract}
The invariant chain (Ii) plays an important role as a chaperone for MHC II maturation and facilitates antigen presentation in vertebrates. We cloned, characterized and made a homology analysis of healthy adult muscovy duck Ii (MDIi), from a poultry farm in the suburban district of Hefei city in China, by rapid amplification of cDNA ends (RACE)-PCR and by measuring expression of the $M D I i$ gene in various tissues by real-time quantitative PCR. A full-length cDNA sequence of MDIi was obtained, 1188-bp long, encoding a 222-amino acid protein. A comparison of the amino acid sequence of Ii between muscovy duck and other birds showed high similarity (66.3-95.3\%). Characteristic functional domains found in Ii of other species, such as cytoplasmic domain, transmembrane domain, class IIassociated Ii-derived peptide (CLIP) and trimerization domain, were identified in MDIi. Although all functional domains of Ii were found to be highly conserved, small differences in the CLIP sequence occur among the various species. Expression of $M D I i$ was detected in all tissues at different levels. A higher expression level was found in the spleen, intestinal mucosa and the bursa stipe (bursa of Fabricius stipe)
\end{abstract}


than other tissues. This tissue-specific expression suggests that MDIi plays an essential role in all tissues and differential expression may be a function of the innate structures and essential functions of these tissues.

Key words: Clone; Differential expression; Invariant chain; RT-PCR; Muscovy duck; RACE

\section{INTRODUCTION}

Major histocompatibility complex class II (MHC II) association invariant chain (Ii, also named CD74) is a conservative type-II transmembrane glycoprotein (Claesson and Peterson, 1983; Strubin et al., 1984). Alternative initiation of translation and differential splicing of the transcription products from a single gene generate four isoforms in humans (O'Sullivan et al., 1987), but only two isoforms in mice (Lennon-Dumenil et al., 2001) and bird (Zhong et al., 2006). Ii is mainly expressed on the surface of specialized antigen-presenting cells (Bryant and Ploegh, 2004), as well as on tumor cells (Voutsas et al., 2007; Holmes et al., 2008). The Ii contains the following functional domains: i) a short amino-terminal cytoplasmic domain; ii) a single transmembrane (TM) domain; iii) a large carboxy-terminal luminal region, which has a class II-associated Ii-derived peptide (CLIP) domain, and a trimerization (TRIM) domain; iv) Ii may also have a thyroglobulin type I domain (Lipp and Dobberstein, 1986; Bremnes et al., 1994, 2000; Silva et al., 2007; Xu et al., 2008).

Ii plays an important role as a chaperone for MHC II protein expression and facilitates antigen presentation by blocking the MHC II peptide binding groove in endoplasmic reticulum and regulating the loading of MHC II molecules with peptides (Denzin and Cresswell, 1995; Ghosh et al, 1995; Cresswell, 1996; Pieters, 1997; Busch et al., 2005). The interaction mechanisms between Ii and MHC II have been further studied in mammal and human diseases such as infection, allergy and tumors. Based on the immune carrier characteristic of Ii presented in the interaction with MHC II (Adams et al., 1997; Nagata et al., 2001), some genetic vaccines encoding an Ii protein, where the CLIP domain has been replaced by sequences encoding special antigen epitopes, have been produced and show significantly greater epitope-specific CD4 (+) T-cell immune responses than DNA vaccines encoding the Ii alone or antigen epitope alone either in vitro or in vivo (van Bergen et al., 2000; Nagata et al., 2002; Hung et al., 2007; Nagaraj et al., 2008). However, to date Ii has not been reported in the muscovy duck, a waterfowl species widely raised in China. To study the function and evolution of Ii of muscovy duck (MDIi), we report the cloning, characterization, homology analysis, and homology modeling of MDIi by rapid amplification of cDNA ends (RACE)-PCR followed by bioinformatics analysis. To investigate the distribution of MDIi in organism, we also show the differential expression of MDIi in various tissues by real-time relativity quantitative PCR (real-time RQ-PCR). This study will attempt to help broaden our knowledge of poultry immunology and could eventually lead to measures to protect poultry from disease.

\section{MATERIAL AND METHODS}

\section{Muscovy duck and sampling}

Healthy adult muscovy ducks at approximately 5 months of age were purchased from 
a poultry farm in the suburban district of Hefei city, China. Samples of blood, brain, bursa fundus (bursa of Fabricius fundus), bursa stipe (bursa of Fabricius stipe), heart, intestinal mucosa, kidney, liver, lung, skeletal muscle, spleen, and thymus were collected and kept at $-80^{\circ} \mathrm{C}$ until use.

\section{RNA isolation and cDNA synthesis}

Total RNA was extracted from tissues using RNAiso Plus (TaKaRa Biotechnology Co., Dalian, China) and single-stranded cDNA from $5 \mu \mathrm{g}$ RNA was synthesized using TaKaRa PrimeScript $^{\mathrm{TM}}$ (TaKaRa) 1st-Strand cDNA Synthesis kit according to manufacturer instructions.

\section{Degenerate PCR}

A pair of degenerate primers, Ii-D-F and Ii-D-R (Table 1 and Figure 1), was designed within the conserved sequences of Ii genes among the selected birds (GenBank accession Nos.: duck, AY905540; goose, HM208131; chicken, AY597053) using the DNAMAN version 6.0 software (Lynnon BioSoft, USA). The primers were used to amplify the MDIi cDNA fragment (called the degenerate fragment) with Taq DNA polymerase (TaKaRa). Amplification products were purified from 1\% agarose gels using a gel extraction kit (BioTek Instruments, Inc., Winooski, VT, USA). The purified fragment was cloned into the pMD-18T vector (TaKaRa) and sent to Sangon Biotech Co. Ltd. (Shanghai, China) for sequencing.

\begin{tabular}{lll} 
Table 1. Primers used in this study. & \\
\hline Application & Primer & Sequence (5' to 3') \\
\hline Degenerate PCR & Ii-D-F & (G/T)TCCATCCTGGTG(A/G)C(C/T)CTGCT \\
& Ii-D-R & CAG(C/G)AGCCA(A/C)T(G/T)GTGCACCA \\
5'-RACE-PCR & 5'-Outer-GSP & CCAATTGTGCATCCAGGACTC \\
& 5'-Outer-primer & CATGGCTACATGCTGACAGCCTA \\
& 5'-Inner-GSP & CCTGACTTGGTCCTCGGTCTTGTTG \\
3'-RACE-PCR & 5'-Inner-primer & CGCGGATCCACAGCCTACTGATGATCAGTCGATG \\
& 3'-Outer-GSP & AGCAACAAGACCGAGGACCAAG \\
& 3'-Outer-primer & TACCGTCGTTCCACTAGTGATTT \\
Full-length PCR & 3'-Inner-GSP & GCCTGCTGGACAACCTCAAGA \\
ORF PCR & Full-Ii-F & CGCGGATCCTCCACTAGTGATTTCACTATAGG \\
& Full-Ii-R & TGGGGCAGCCATGGCCGAGGAG \\
Real-time PCR & ORF-F & TCCTTCACACAACGCCCTCACT \\
& ORF-R & TGGGGCAGCCATGGCCGAGGAG \\
& RTP-F & CCTCTTCTACTTGGCTTCTCA \\
RTP-R & GCAACAAGACCGAGGACCAAG \\
& $\beta$-actin-F & CTTCAGGCTCTTGAGGTTGTC \\
& $\beta$-actin-R & ATGTGGATCAGCAAGCAGGA \\
\hline
\end{tabular}

RACE $=$ rapid amplification of cDNA ends; Full-length PCR $=$ PCR for obtaining the fragments including most partial sequence of full-length invariant chain of muscovy duck (MDIi) cDNA; ORF PCR = PCR for obtaining the fragments including the open reading frame of MDIi cDNA; $F=$ forward primer; $\mathrm{R}=$ reverse primer; $\mathrm{GSP}=$ gene special primer.

\section{RACE-PCR}

RACE was used to obtain the 5'- and 3'-end sequences for isolating full-length cDNA of MDIi genes. Four specific nested primers (5'-Outer-GSP and 5'-Inner-GSP, 3'-Outer-GSP 
and 3'-Inner-GSP; Table 1 and Figure 1) were designed according to degenerate fragment sequences. 5'-Outer-primer and 5'-Inner-primer, 3'-Outer-primer and 3'-Inner-primer (Table 1 and Figure 1) were in the corresponding RACE Kit (TaKaRa). 5'-Outer-GSP and 5'-Outerprimer, 5'-Inner-GSP and 5'-Inner-primer were used for amplification of the 5'-end of MDIi cDNA. 3'-Outer-primer and 3'-Outer-GSP, 3'-Inner-primer and 3'-Inner-GSP were used for amplification of the 3'-end of MDIi cDNA. 5'-RACE and 3'-RACE were carried out using the 5'-Full RACE Kit (TaKaRa) and the 3'-Full RACE Core Set Ver. 2.0 (TaKaRa) according to manufacturer instructions. The products of 3'- and 5'-RACE were purified and cloned into the pMD-18T vector and sent to the Sangon Shanghai Biotechnology Co., for sequencing.

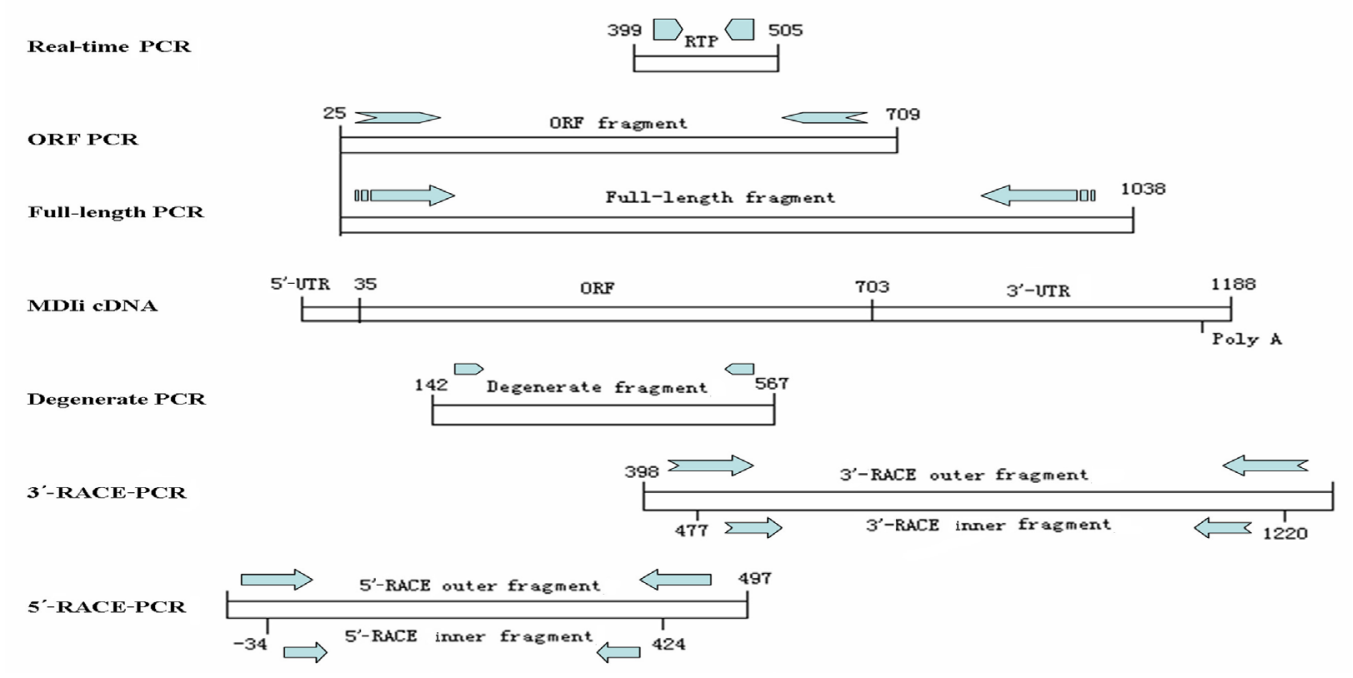

Figure 1. Relative position of the primer pairs used for obtaining the fragment sequences of invariant chain of muscovy duck (MDIi) cDNA. Each primer pair was shown by two arrows of the same shape in opposite direction.

\section{Sequence being assembled and verified}

The deduced full-length MDIi cDNA sequence was assembled using the degenerate fragment sequences, the 3'- and 5'-RACE products using the DNAMAN software (Lynnon BioSoft). To confirm the assembled full-length MDIi cDNA and the complete open reading frame (ORF) sequence, the full-length primer pair (Full-Ii-F and Full-Ii-R; Table 1 and Figure 1) and the ORF primer pair (ORF-F and ORF-R; Table 1 and Figure 1) were designed according to the assembled full-length MDIi cDNA. Then, the assembled sequence was verified by sequencing the fragment amplified from the primer pairs Full-Ii-F and Full-Ii-R and subjected to homology analysis. The ORF was also verified by ORF PCR and sequencing using the primers ORF-F and ORF-R.

\section{Bioinformatics analysis and homology modeling}

The cDNA sequences were analyzed by DNASTAR 5.0 (DNASTAR Inc., Madison, 
WI, USA). Similarity analysis was performed with the BLAST algorithm at NCBI (http:// www.ncbi.nlm.nih.gov/BLAST) and the DNAMAN version 6.0 software. The putative domains, signal sequence and motif prediction were identified by the Simple Modular Architecture Research Tool (SMART, http://smart.embl-heidelberg.de), the ScanProsite programs in the PROSITE database (http://ca.expasy.org/prosite) and Motif Scan (http://hits.isb-sib.ch/ cgi-bin/PFSCAN), respectively. Multiple alignments of the Ii proteins were performed with the Clustal W multiple alignment programs (http://www.ebi.ac.uk/clustalw/). The neighborjoining phylogenetic tree was constructed using MEGA 4.0 (http://www.megasoftware.net/ mega). The structure model of Ii was obtained using the Expasy's translate tool (http://www. expasy.org/). The theoretical models were displayed and analyzed with the RasMol software (http://www.RasMol.org).

\section{Real-time RQ-PCR}

The differential expression of MDIi in various tissues was detected by real-time RQPCR with the primer pair (RTP-F and RTP-R; Table 1 and Figure 1) designed according to the confirmed MDIi cDNA. Also the primer pair ( $\beta$-actin-F and $\beta$-actin-R; Table 1 and Figure 1 ) was designed according to the duck $\beta$-actin gene (GenBank No. GU564232). The cDNA from 12 tissues was generated by 2-stage real-time RT-PCR using PrimeScript RT Master Mix Perfect Real Time (TaKaRa). Finally, cDNA was adjusted to $50 \mathrm{ng} / \mu \mathrm{L}$ and stored at $4{ }^{\circ} \mathrm{C}$ for subsequent fluorescent real-time PCR.

Real-time RQ-PCR was conducted on a 7500 Real-Time PCR system (Applied Biosystems, Carlsbad, CA, USA) with the primers RTP-F and RTP-R for amplifying the Ii fragment. Amplifications were carried out at a final volume of $20 \mu \mathrm{L}$, containing $100 \mathrm{ng}$ cDNA sample $(2 \mu \mathrm{L} \times 50 \mathrm{ng} / \mu \mathrm{L}), 10 \mu \mathrm{L}$ SYBR Premix Ex Taq ${ }^{\mathrm{TM}}$ II $(2 \mathrm{X})$ (TaKaRa), $0.4 \mathrm{~mL}$ ROX Reference Dye II (50X), $0.4 \mu \mathrm{L}$ of each primer $(10 \mu \mathrm{M})$ and $6.8 \mu \mathrm{L} \mathrm{ddH}_{2} \mathrm{O}$. PCR amplification was performed in triplicate per tissue, under the following conditions: $30 \mathrm{~s}$ at $95^{\circ} \mathrm{C}$, followed by 40 cycles consisting of $5 \mathrm{~s}$ at $95^{\circ} \mathrm{C}$ and $34 \mathrm{~s}$ at $60^{\circ} \mathrm{C}$. $\beta$-actin was used as internal control. Expression of MDIi in the tissue, which had the lowest value of expression level, was used as calibrator. ANOVA was used for statistical analysis. $\mathrm{P}<0.01$ indicates statistical significance.

\section{RESULTS}

\section{Conservation of MDIi cDNA sequence}

A degenerate fragment of $426 \mathrm{bp}$ was first amplified from total RNA of the muscovy duck (Figure 1). Then, a 744-bp 3'-RACE fragment and a 459-bp 5'-RACE fragment were amplified separately (Figure 1). The above fragments were verified as partial fragments of Ii by NCBI blast analysis. After splicing, assembly and analysis on the above fragments, a deduced full-length MDIi was obtained and verified to be true by full-length PCR and ORF PCR (Figure 1). The full-length MDIi cDNA (GenBank accession No. HQ909102) was 1188 bp, including a 669-bp ORF located between nucleotides 35 and 703, a 34-bp 5'-terminal untranslated region (5'-UTR) and a 485-bp 3'-UTR followed by a 12-bp poly(A) tail.

The full-length MDIi cDNA sequence of the muscovy duck was compared with that of other birds, mice and human in homology. The ORF, 3'-UTR of MDIi were also compared 
with those of other Ii species (Figure 2). The data were not shown on the 5'-UTR because it was too short to reflect the homology among the species listed. The cDNA sequence of MDIi showed high conservation, sharing $62.7-95.6 \%$ identity with other bird Ii and even showing similarity with mouse Ii (48.7\%) and human Ii (49.2\%). The ORF sequences were more conservative than 3'-UTR among Ii of all species listed.

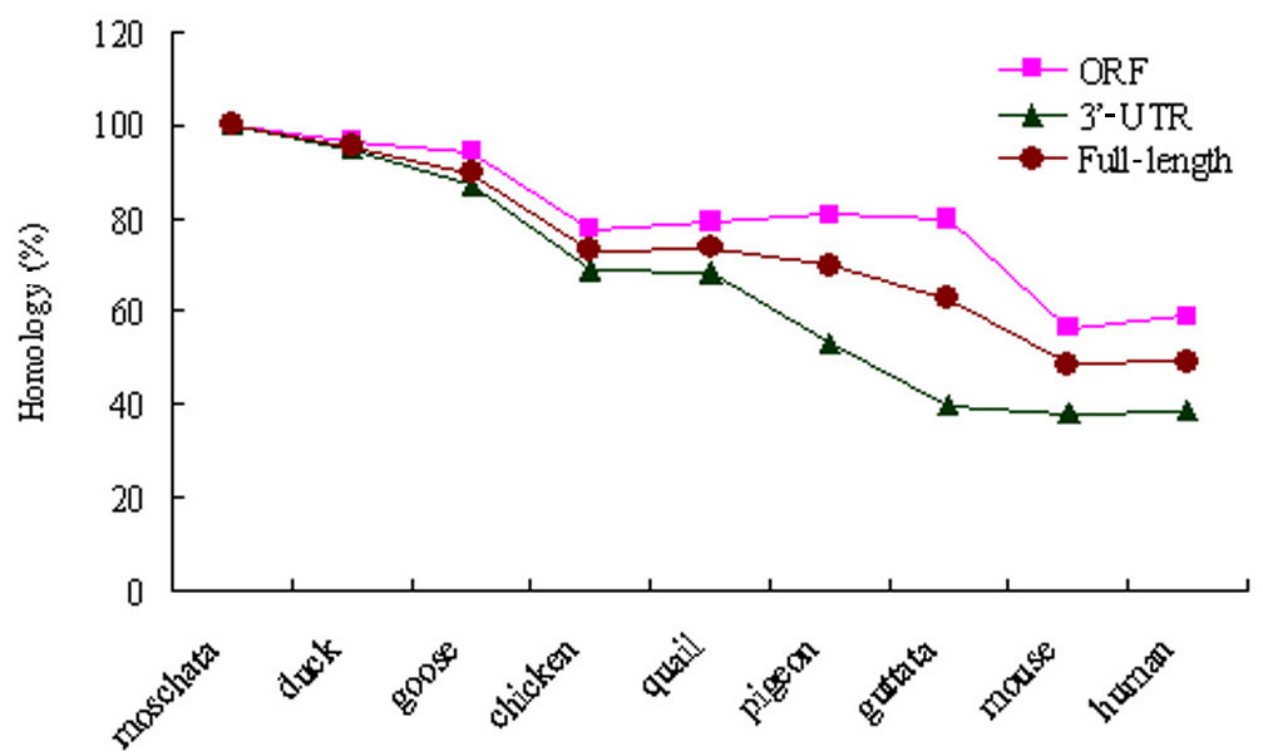

Figure 2. Homology analysis of fragment sequences of invariant chain (Ii) cDNA among various species. The GenBank accession numbers of Ii cDNA sequence are: moschata (muscovy duck), HQ909102; duck, AY905540; goose, HM208131; chicken, AY597053; quail, HM011049; pigeon, AY904337; guttata, DQ215319; mice, NM_010545; human, X00497.1. The ORF, 3'-UTR and full-length denote open reading frame, 3'-terminal untranslated region and full-length cDNA sequence of Ii, respectively. The Y-axis represents the identity of corresponding fragment sequence of Ii cDNA between muscovy duck and other species.

\section{Diversity in CLIP domains of conservative Ii among various species}

The ORF of MDIi cDNA encodes a protein consisting of 222 amino acids (Figure 3), with a predicted molecular mass of $24.68 \mathrm{kDa}$, a theoretical $\mathrm{pI}$ of 9.19 and a charge of 4.26. All the characteristic functional domains found in Ii of other species, such as cytoplasmic domain, TM domain, CLIP domain, and TRIM domain, were identified in MDIi by SMART (Figure 3). The MHC II interaction region, which was required for association with MHC II in the MHC II processing pathway, started at position 1 and ended at position 113 in the amino acid sequence of MDIi. This region contained a cytoplasmic domain, a single-TM domain and a CLIP domain. The locations of the functional domains in the amino acid sequence of MDIi are shown in Figure 3. A potential N-glycosylation site at 123-126 was found in the MDIi protein by ScanProsite (Applied Biosystems) programs, but no signal peptide was found using the Motif Scan software. Homology analysis (Figure 4) demonstrated that the amino acid sequence of MDIi shared remarkably high homology (66.3-95.3\%) compared with that of 
other birds, yet lower homology (about 40\%) with Ii of mice and human. The homologies of different domains of MDIi with corresponding domains of other Ii species are also shown in Figure 4. The TM domains displayed the highest conservation, sharing $60.7-100 \%$ identity among the Ii listed, and the cytoplasmic domains were the next. Although the CLIP domains revealed slight diversity, they still maintain over 50\% identity. The TRIM domains displayed coincident conservation with the full-length Ii listed.

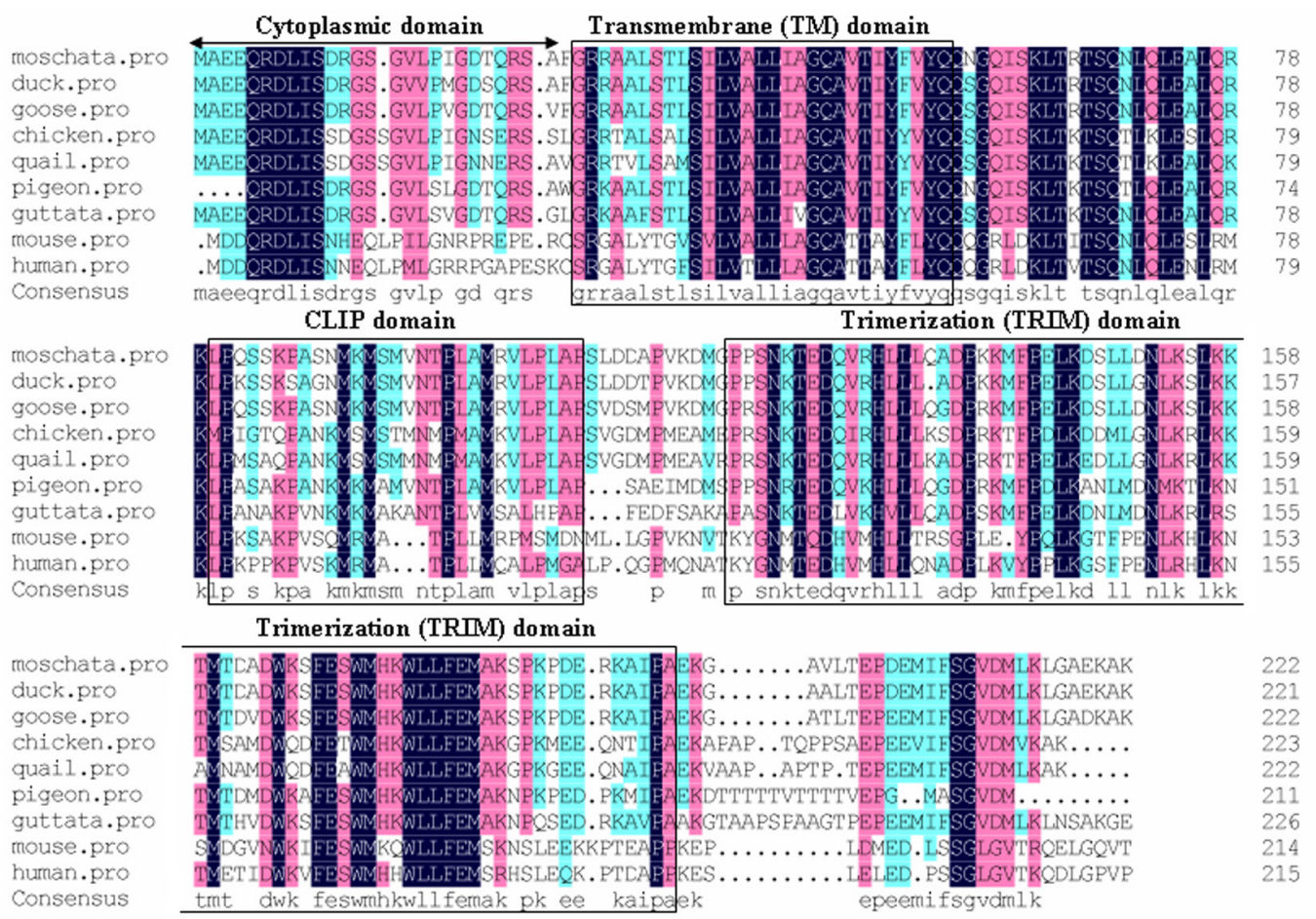

Figure 3. Multiple alignments of amino acid sequences of the invariant chain (Ii) protein among various species. The GenBank accession numbers of the amino acid sequences of Ii proteins among various species are: moschata, MDIi (Ii of muscovy duck); duck, AAX89536; goose, ADK11994; chicken, AAT36345; quail, ADG01642; pigeon, AAX47311; guttata, ACH44981; mouse, NP_034675; human, CAA25193. The functional domain of Ii was characterized and named above the corresponding sequences, which are marked by a black rectangle box. Amino acid sequences of Ii proteins were compared through domain-by-domain among various species. CLIP $=$ class IIassociated Ii-derived peptide domain.

\section{Similar frequency of amino acid in Ii among various species}

The phylogenetic tree (Figure 5) showed that birds and mammals were divided into two evolutionary clusters, a bird cluster and a mammal cluster. MDIi was more closely related to Ii of duck (95.3\%) and goose (93.8\%) than others (Figure 4). In the bird cluster, the divergence of amino acids in Ii of 7 birds was compared and 19 kinds of amino acids besides Cys were detected in each Ii with MEGA 4.0. The 19 kinds of amino acids were coincident at similar average frequency in each Ii. Among the 19 kinds of amino acids in MDIi, the six kinds 
of amino acids Leu (13.06\%), Lys (9.46), Ala (9.01\%), Ser (8.53\%), Pro (6.76\%), and Asp $(6.76 \%)$ were found at higher rates than the others. Total rates of the six kinds of amino acids of MDIi were $54 \%$, thus the six kinds of amino acids were the mainly components of MDIi.

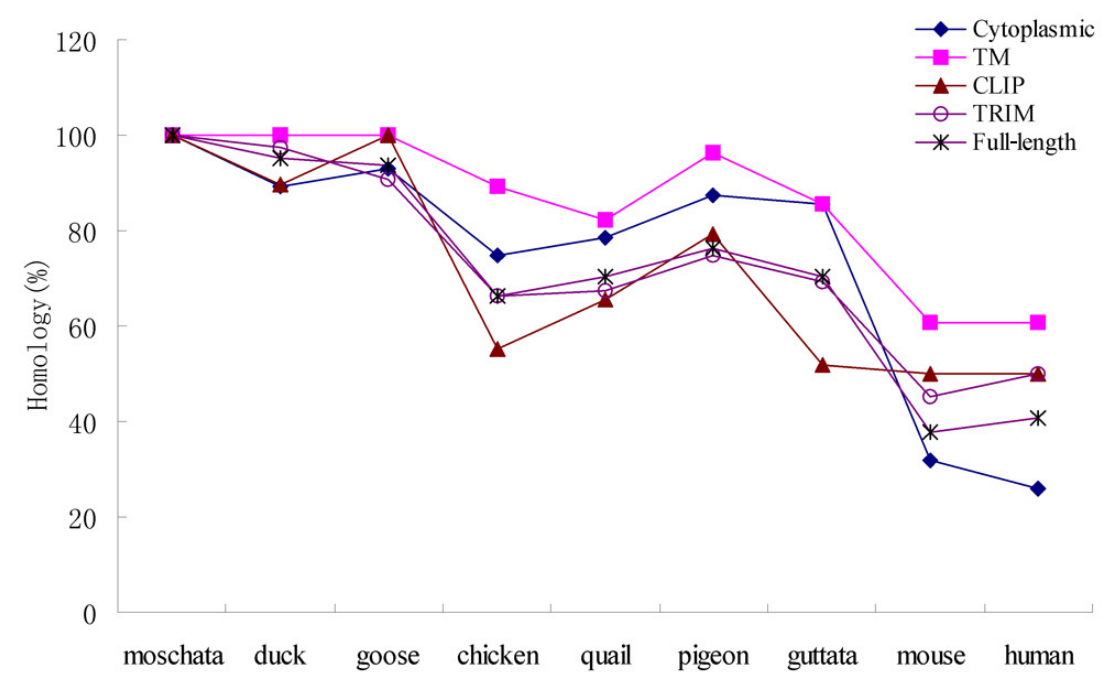

Figure 4. Homology analysis of the functional domains of invariant chain (Ii) proteins among birds, mice and human. The GenBank accession numbers are listed in Figure 3. Here, cytoplasmic, TM, CLIP, TRIM, and fulllength denote the cytoplasmic domain, transmembrane domain, class II-associated Ii-derived peptide domain, trimerization domain, and the full-length sequence of the Ii protein, respectively. The value at each node represents the identity of corresponding amino acid sequences of Ii between muscovy duck and other species.

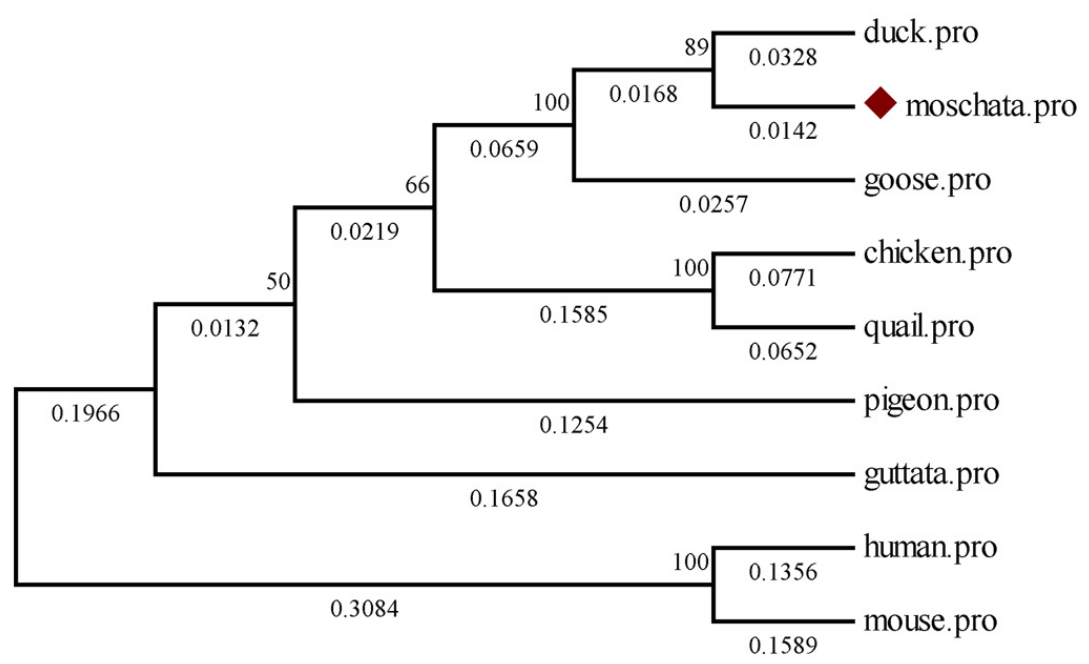

Figure 5. Phylogenetic tree of Ii proteins among various species created by the MEGA 4.0 software with the neighbor-joining method based on amino acid sequence references listed in Figure 3. Numbers under each branch line indicate the evolutional distance assessed by 1000 bootstrap repetitions with confidence values above $50 \%$ presented on nodes. 


\section{Conservation in structure models of Ii among various species}

The three-dimensional (3-D) structure models of partial TRIM domain of Ii among six species (Figure 6) showed that the backbone of Ii in each model was remarkably similar and there were 3 similar $\alpha$-helix in all models. Among the $3 \alpha$-helix, two turns were formed with similar amino acids. For instance, Met (Met 160 in muscovy duck, Met 159 in duck, Met 160 in goose, Met 161 in chicken, Met 155 in mice, and Met 157 in human) were found in the first turn of each model. In the second turns of all models, there were same amino acids (e.g., Pro, Lys, Asp, Ser, et al.) in the corresponding site. However, more different amino acids were found in the second turns than in the first ones. Interestingly, the different amino acids at key sites of each model did not disturb the framework of Ii.
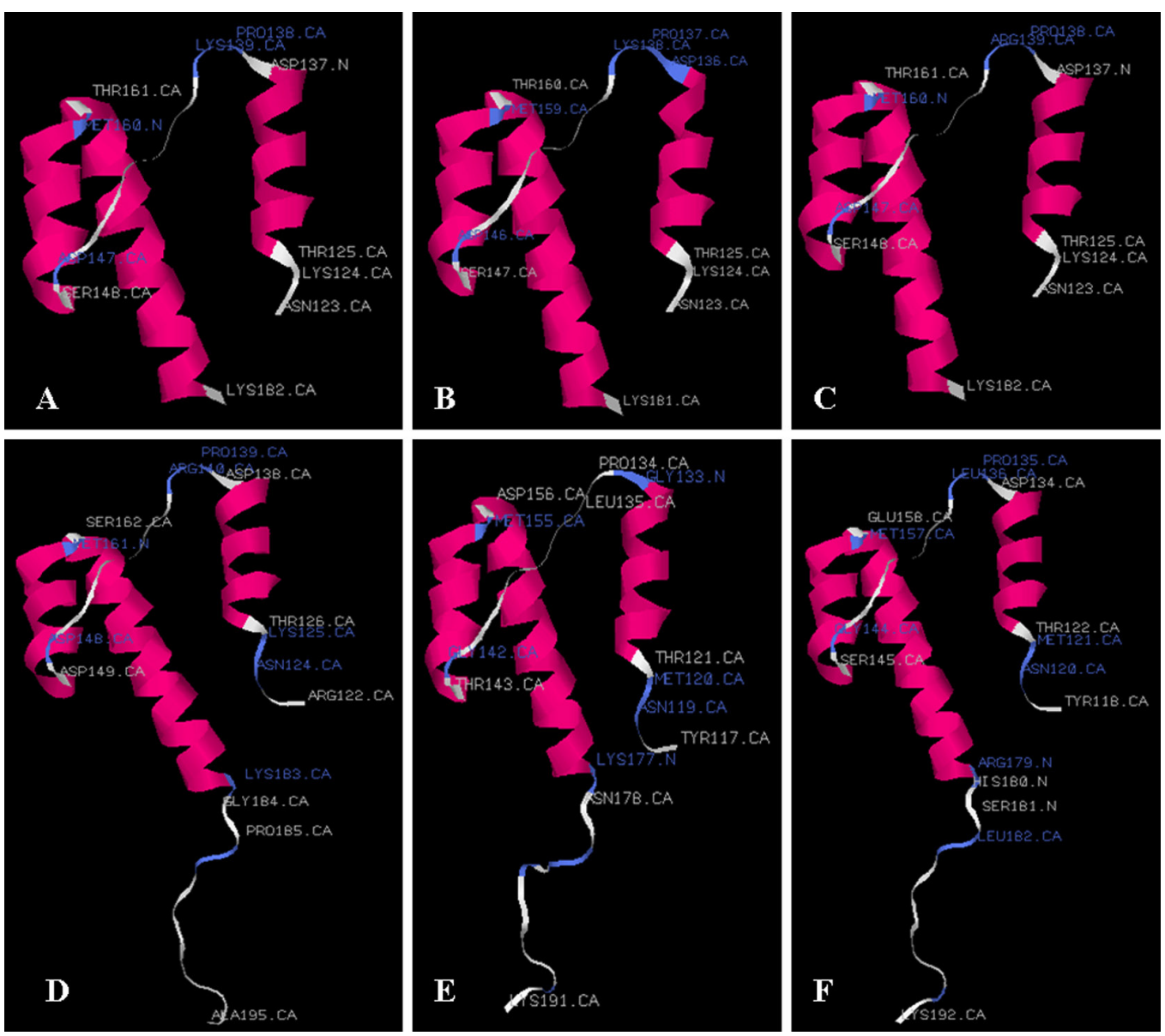

Figure 6. Three-dimensional (3-D) structure models of partial trimerization region of six Ii species of which the amino acid sequence references are listed in Figure 3. The 3-D models of the Ii protein in muscovy duck (A), duck (B), goose (C), chicken (D), mice (E), and human (F) were generated on template 1iieA (99.9 A) of human Ii using Swiss-Model sever and viewed by the RasMol software. Amino acid residues at key sites of models are marked individually. 


\section{MDIi gene expressing in all tissues tested at different levels}

The expression of MDIi was detected in all tissues, but their expression levels were tissue-specific (Figure 7). The highest expression of MDIi was found in spleen, 1.65-fold over the calibrator, and the intestinal mucosa, which is not a traditional immune tissue, exceeding 1.54-fold versus the calibrator. Next to them, bursa stipe, blood, liver, and kidney abundantly expressed MDIi, all exceeding 0.85 -fold versus the calibrator. Interestingly, distinct expression of MDIi was found in two parts of bursa of Fabricius. Bursa stipe expressed MDIi 1.11fold higher than the bursa fundus. In contrast, the expression of MDIi reduced dramatically in the thymus, lung, heart, and muscle at an almost similar expression level about 0.5 -fold over the calibrator. In addition, the brain had the second lowest level of MDIi expression next to the bursa fundus.

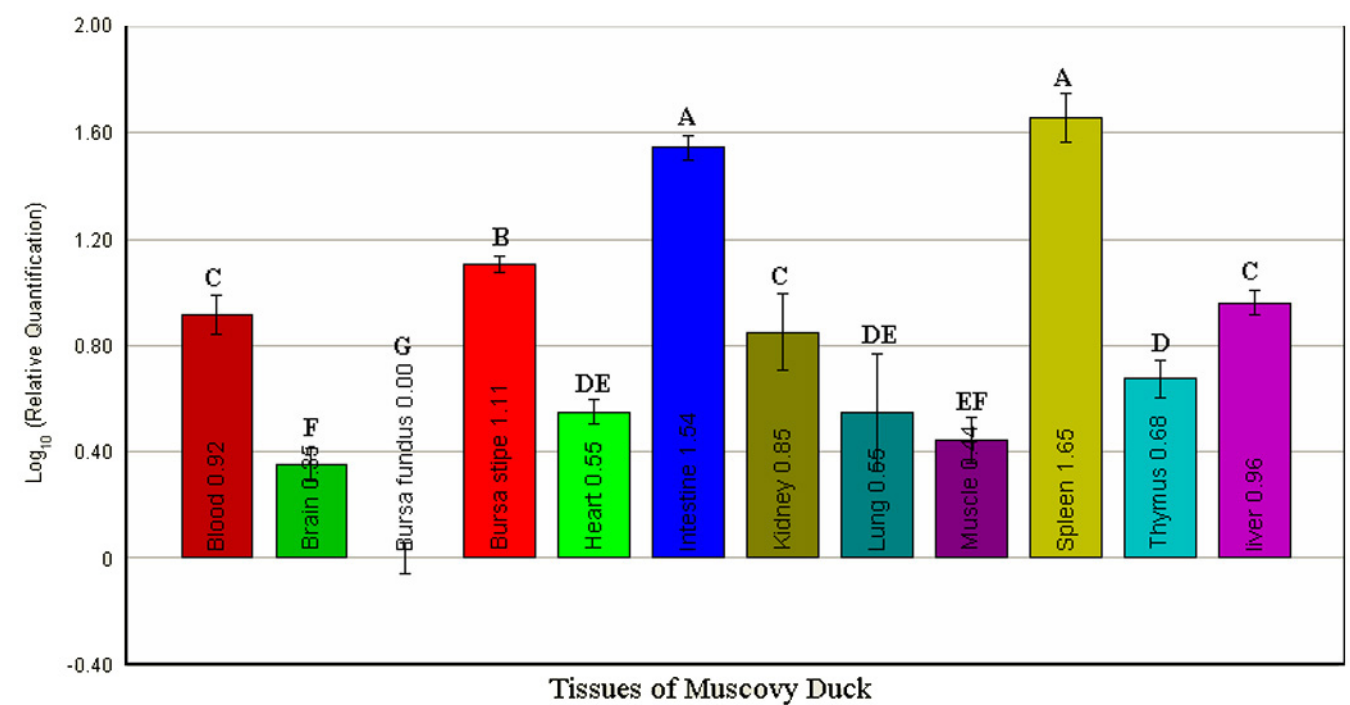

Figure 7. Expression profile of MDIi in various tissues from the muscovy duck. The expression levels are measured by real-time RQ-PCR in samples from blood, brain, bursa fundus, bursa stipe, heart, intestine mucosa, kidney, lung, muscle, spleen, thymus, and liver from the muscovy duck at about 5 months of age. MDIi gene expression is normalized to endogenous control $\beta$-actin. MDIi expression in bursa fundus serves as calibrator. MDIi gene expression levels are achieved using the $2^{-\Delta \Delta C t}$ method of quantification. The relative expression level of MDIi in variant tissues is shown as the mean of three measurements and analyzed by one-way ANOVA. $\mathrm{P}<0.01$ is denoted with capital letter above each bar. Groups marked with the same letters are not statistically different.

\section{DISCUSSION}

\section{The structural conservation under evolution pressure}

In this study, we found that the MDIi protein showed high similarity of full-length amino acid sequences with other Ii species (Figure 4), with a similar format of domain structure of Ii among various species. The cytoplasmic, TM, CLIP and TRIM domains identified 
in MDIi were coincident with those of other Ii protein species (Figure 3). These domains of MDIi show high identities with corresponding parts of Ii among other species as well. Although the CLIP domain revealed slight diversity, it still maintained over 50\% identity. The conservation of Ii is also evidenced in similar amino acid frequency in Ii of each species, so that the backbone in 3-D models of Ii in various species is remarkably similar. Furthermore, the framework in each model of Ii was not disturbed in spite of the amino acid difference in the key site of each model (Figure 6). Moreover, the conservation is revealed in the structure models of Ii in various species as well. Therefore, the MDIi gene might be derived from common progenitor gene with the Ii gene of other species. Also the high conservation of Ii in various species might result in similar functions. In this sense, muscovy duck may serve as a useful research animal model in the future.

Although Ii is highly conservative in various species, the structural alteration of Ii in the process of phylogeny dose exists among them, similar to the slight diversity in CLIP among various species. Although the alteration of CLIP is not alone in evolutionary history, there may be coevolution occurring with other immune molecules, such as the pleomorphic MHC II. The CLIP domain is essential for MHC II folding, transport, and peptide occupancy. Nevertheless, the MHC II molecule has a high degree of polymorphism, especially in the groove, which is bound by the CLIP. This diversity of MHC II is believed to be driven by selection for resistance to pathogens (Bernatchez and Landry, 2003; Surridge et al., 2008; Meyer-Lucht et al., 2010). The CLIP might be modified to fit the variant MHC II under the selection pressure. Therefore, the alteration of CLIP may be a selective advantage by mutating under environmental pressure, especially under the various disease pressures in evolutionary history (Marchalonis et al., 2002).

\section{Tissue-specific expression of MDIi is related to structure and function of tissues}

In this study, MDIi was extensively expressed in various tissues at different levels. The patterns of MDIi expression in various tissues in the muscovy duck were coordinate with that in chicken (Zhong et al., 2004), duck (Zhong et al., 2006) and mammals (Koch and Harris, 1984). This demonstrates that MDIi is necessary in all tissues. Why tissuespecific expression? We find that tissue-specific expression of MDIi may be related to the innate structures and essential functions of these tissues. As conventional immune organs in which there are lymphoid tissues, spleen and bursa stipe highly express MDIi but thymus expresses MDIi at a low level. Difference of MDIi levels in these immune tissues may be closely related to their structural change accompanying body up-growth. Because the muscovy ducks used in this study were approaching adulthood, the spleen was fully developed to be the main immune organ. Thus, the highest level expression of MDIi in spleen was detected. However, the structure of bursa stipe and thymus gradually degenerate accompanying up-growth to adulthood, so that the functions of bursa stipe and thymus are evidently down-regulated, with thymus being down-regulated faster than bursa stipe. Interestingly, bursa fundus expresses MDIi at a very low level. This may be the result of the structural differences between bursa stipe and bursa fundus. The bursa stipe is the T-dependent area, which first appears after hatching and grows rapidly to reach adulthood in the second week of postnatal life, yet rare lymphoid tissues are found in the bursa fundus (Cortes et al., 1995; Zhang et al., 2000; Qi and Chen, 2006). The differential expression of Ii in different parts 
of the bursa of Fabricius is consistent with histological and immunological studies, which reveal distinctions among different parts of bursa of Fabricius. Intestinal mucosa expresses MDIi at the second high level. We can speculate that the epithelial cells of intestinal mucosa (IEC) are the major contributor of the MDIi expression. This notion is supported by studies on human mucosal immunity demonstrating that the Ii expressed in IEC plays an important role as potential regulators of local immune responses (Barrera et al., 1998; Hershberg and Mayer, 2000). Analogously, it has been reported that gastric epithelial cells and colon epithelial cells have a high expression level of surface Ii, which is closely correlated with anti-tumor immunity (Barrera et al., 2005; Tamori et al., 2005; Maharshak et al., 2010). IEC are located at a strategic position between the external environment and the most extended lymphoid tissue in the body. They provide antigenic information to the immune system and are involved in the balance tolerance/allergy to food antigens in addition to their central role in the absorption of nutrients (Mallegol et al., 2005). Therefore, the role of Ii in mucosal immunity is very important for the integrity and function of digestive tract. Liver, kidney and blood, which are highly active in metabolism, also abundantly express MDIi, implying that there may be immune events involved in the course of metabolism. The brain belongs to the central nervous system where there is a hematoencephalic barrier to protect it against external antigen in order to safely perform neuroregulation or decision control. Although immunity is not the main function of brain, lower level of Ii, possibly expressed by microglia cells, may help protect the brain tissue.

Therefore, the body not only has conventional immune organs or tissues such as spleen, bursa of Fabricius, thymus, and blood, but also has untraditional ones, just like intestinal mucosa, liver, and kidney in the muscovy duck. Expression of MDIi in these tissues suggests that some cells may function as immune cells to collaborate with the immune system to protect the tissues. Moreover, expression of MDIi relies on the innate structures and essential functions of tissues by some regulatory mechanism, so that the MDIi expression level is tissue-specific. However, the regulatory mechanism of MDIi expression in different tissue remains yet to be investigated. Future investigation may focus on this area of study.

\section{CONCLUSION}

We cloned a 1188-bp cDNA sequence of MDIi encoding a protein of 222 amino acids. The MDIi shows high conservation compared with Ii of other species. Among conservative functional domains of Ii among various species, the CLIP domain shares a slight diversity, which may be a selective advantage under the selective pressure of the environment in the process of phylogeny. MDIi can be detected in all tissues tested at different levels, suggesting that MDIi may play an essential role in various tissues and tissue-specific expression may be related to the innate structures and essential functions of tissues.

\section{ACKNOWLEDGMENTS}

Research supported by the grant from the National Natural Science Foundation of China (\#30671537) and the grant from the Teaching Research Projects of Anhui Department of Education (\#2008jyxm460). 


\section{REFERENCES}

Adams S, Albericio F, Alsina J, Smith ER, et al. (1997). Biological activity and therapeutic potential of homologs of an Ii peptide which regulates antigenic peptide binding to cell surface MHC class II molecules. Arzneimittelforschung 47: 1069-1077.

Barrera CA, Almanza RJ, Ogra PL and Reyes VE (1998). The role of the invariant chain in mucosal immunity. Int. Arch. Allergy Immunol. 117: 85-93.

Barrera CA, Beswick EJ, Sierra JC, Bland D, et al. (2005). Polarized expression of CD74 by gastric epithelial cells. $J$. Histochem. Cytochem. 53: 1481-1489.

Bernatchez L and Landry C (2003). MHC studies in nonmodel vertebrates: what have we learned about natural selection in 15 years? J. Evol. Biol. 16: 363-377.

Bremnes B, Madsen T, Gedde-Dahl M and Bakke O (1994). An LI and ML motif in the cytoplasmic tail of the MHCassociated invariant chain mediate rapid internalization. J. Cell Sci. 107 ( Pt 7): 2021-2032.

Bremnes B, Rode M, Gedde-Dahl M, Nordeng TW, et al. (2000). The MHC class II-associated chicken invariant chain shares functional properties with its mammalian homologs. Exp. Cell Res. 259: 360-369.

Bryant P and Ploegh H (2004). Class II MHC peptide loading by the professionals. Curr. Opin. Immunol. 16: 96-102.

Busch R, Rinderknecht CH, Roh S, Lee AW, et al. (2005). Achieving stability through editing and chaperoning: regulation of MHC class II peptide binding and expression. Immunol. Rev. 207: 242-260.

Claesson L and Peterson PA (1983). Association of human gamma chain with class II transplantation antigens during intracellular transport. Biochemistry 22: 3206-3213.

Cortes A, Fonfria J, Vicente A, Varas A, et al. (1995). T-dependent areas in the chicken bursa of Fabricius: an immunohistological study. Anat. Rec. 242: 91-95.

Cresswell P (1996). Invariant chain structure and MHC class II function. Cell 84: 505-507.

Denzin LK and Cresswell P (1995). HLA-DM induces CLIP dissociation from MHC class II alpha beta dimers and facilitates peptide loading. Cell 82: 155-165.

Ghosh P, Amaya M, Mellins E and Wiley DC (1995). The structure of an intermediate in class II MHC maturation: CLIP bound to HLA-DR3. Nature 378: 457-462.

Hershberg RM and Mayer LF (2000). Antigen processing and presentation by intestinal epithelial cells - polarity and complexity. Immunol. Today 21: 123-128.

Holmes JP, Benavides LC, Gates JD, Carmichael MG, et al. (2008). Results of the first phase I clinical trial of the novel II-key hybrid preventive HER-2/neu peptide (AE37) vaccine. J. Clin. Oncol. 26: 3426-3433.

Hung CF, Tsai YC, He L and Wu TC (2007). DNA vaccines encoding Ii-PADRE generates potent PADRE-specific CD4+ T-cell immune responses and enhances vaccine potency. Mol. Ther. 15: 1211-1219.

Koch N and Harris AW (1984). Differential expression of the invariant chain in mouse tumor cells: relationship to B lymphoid development. J. Immunol. 132: 12-15.

Lennon-Dumenil AM, Roberts RA, Valentijn K, Driessen C, et al. (2001). The p41 isoform of invariant chain is a chaperone for cathepsin L. EMBO J. 20: 4055-4064.

Lipp J and Dobberstein B (1986). The membrane-spanning segment of invariant chain (I gamma) contains a potentially cleavable signal sequence. Cell 46: 1103-1112.

Maharshak N, Cohen S, Lantner F, Hart G, et al. (2010). CD74 is a survival receptor on colon epithelial cells. World J. Gastroenterol. 16: 3258-3266.

Mallegol J, van Niel G and Heyman M (2005). Phenotypic and functional characterization of intestinal epithelial exosomes. Blood Cells Mol. Dis. 35: 11-16.

Marchalonis JJ, Kaveri S, Lacroix-Desmazes S and Kazatchkine MD (2002). Natural recognition repertoire and the evolutionary emergence of the combinatorial immune system. FASEB J. 16: 842-848.

Meyer-Lucht Y, Otten C, Püttker T, Pardini R, et al. (2010). Variety matters: adaptive genetic diversity and parasite load in two mouse opossums from the Brazilian Atlantic forest. Conserv. Genet. 11: 2001-2013.

Nagaraj S, Neumann J, Winzen B, Frank S, et al. (2008). Pancreas carcinoma antigen fused to invariant chain elicits T-cell response and tumor growth inhibition. Pancreas 37: 321-327.

Nagata T, Higashi T, Aoshi T, Suzuki M, et al. (2001). Immunization with plasmid DNA encoding MHC class II binding peptide/CLIP-replaced invariant chain (Ii) induces specific helper T cells in vivo: the assessment of Ii p31 and p41 isoforms as vehicles for immunization. Vaccine 20: 105-114.

Nagata T, Aoshi T, Suzuki M, Uchijima M, et al. (2002). Induction of protective immunity to Listeria monocytogenes by immunization with plasmid DNA expressing a helper T-cell epitope that replaces the class II-associated invariant chain peptide of the invariant chain. Infect. Immun. 70: 2676-2680. 
O'Sullivan DM, Noonan D and Quaranta V (1987). Four Ia invariant chain forms derive from a single gene by alternate splicing and alternate initiation of transcription/translation. J. Exp. Med. 166: 444-460.

Pieters J (1997). MHC class II restricted antigen presentation. Curr. Opin. Immunol. 9: 89-96.

Qi BM and Chen XY (2006). Distribution and histology of fabricius' bursa-associated lymphoid tissues (FBALT) in muscovy duck. Fujian J. Agric. Sci. 21: 127-130.

Silva DS, Reis MI, Nascimento DS, do Vale A, et al. (2007). Sea bass (Dicentrarchus labrax) invariant chain and class II major histocompatibility complex: sequencing and structural analysis using 3D homology modelling. Mol. Immunol. 44: 3758-3776

Strubin M, Mach B and Long EO (1984). The complete sequence of the mRNA for the HLA-DR-associated invariant chain reveals a polypeptide with an unusual transmembrane polarity. EMBO J. 3: 869-872.

Surridge AK, van der Loo W, Abrantes J, Carneiro M, et al. (2008). Diversity and evolutionary history of the MHC DQA gene in leporids. Immunogenetics 60: 515-525.

Tamori Y, Tan X, Nakagawa K, Takai E, et al. (2005). Clinical significance of MHC class II-associated invariant chain expression in human gastric carcinoma. Oncol. Rep. 14: 873-877.

van Bergen J, Camps M, Offringa R, Melief CJ, et al. (2000). Superior tumor protection induced by a cellular vaccine carrying a tumor-specific $\mathrm{T}$ helper epitope by genetic exchange of the class II-associated invariant chain peptide. Cancer Res. 60: 6427-6433.

Voutsas IF, Gritzapis AD, Mahaira LG, Salagianni M, et al. (2007). Induction of potent CD4+ T cell-mediated antitumor responses by a helper HER-2/neu peptide linked to the Ii-Key moiety of the invariant chain. Int. J. Cancer 121: 2031-2041.

Xu FZ, Ye H, Wang JJ and Yu WY (2008). The effect of site-directed mutagenesis of the ambient amino acids of leucinebased sorting motifs on the localization of chicken invariant chain. Poult. Sci. 87: 1980-1986.

Zhang JH, Deng SZ, Chen NH, Quyang JH, et al. (2000). Biological effects of the T-cell area of chicken bursa. Acta Agric. Univ. Jiangxiensis 22: 110-112.

Zhong DL, Yu WY, Liu YH, Liu J, et al. (2004). Molecular cloning and expression of two chicken invariant chain isoforms produced by alternative splicing. Immunogenetics 56: 650-656.

Zhong DL, Yu WY, Bao M, Xu ZB, et al. (2006). Molecular cloning and rnRNA expression of duck invariant chain. Vet. Immunol. Immunop. 110: 293-302. 